\title{
An Effective and Active Bandwidth Distribution in Networked Control Systems
}

\author{
M.Sundar Rajan, Abraham Mesfin, Satenaw Sando
}

\begin{abstract}
Networked Control System (NCS) is a method composed of physically shared smart devices that can observe the surroundings, work on it, and converse with one another by means of a communication system to attain a widespread purpose. Characteristic examples that fall into this section are Wireless Sensors and Actuators Networks (WSANs) for ecological analyzing and checking, multi-vehicle systems for composed investigation, camera systems for observation, multicamera facilitated movement catch, shrewd lattices for vitality circulation and the executives, and so forth. NCSs changes from increasingly customary control systems as a result of their interdisciplinary which needs the combination of control hypothesis, correspondences, software engineering and programming designing. Plenty of communication modes are available from telephone lines, cell phone networks, satellite networks and most widely used is internet. The choice of network depends upon the application to be served. Internet is the most suitable and inexpensive choice for many applications where the plant and the controller are far from each other. The troubles present in the structure of control systems that are solid to correspondence parameters like transfer speed, arbitrary deferral and packet loss, to computational parameters in light of the tremendous amount of information to be handled or to the mutual idea of the detecting and control to ongoing execution on limited resources and due to the unpredictability to the huge number of untrustworthy agent present. With the limited measure of data transmission accessible, it is improved to use it ideally and proficiently. This further raises the requirement for need choices issue for controlling a series of actuators for a progression of tasks. The proposed methodology deals broadly made in two distinct directions. The first direction aims at a control theoretical analysis while considering the network as a constant parameter like special controllers and altering the sampling rate. The second direction aims the design of new communication network infrastructures, algorithms or protocols like designing static and dynamic message scheduling algorithms. This method combines both directions and depends on the well- recognized results in both communication networks and control theory.
\end{abstract}

Keywords: Networked Control System (NCS), Controllers, Wireless Sensor and Actuator Network (WSAN), Random delay, Bandwidth, Protocols.

Revised Manuscript Received on April 25, 2020.

* Correspondence Author

Dr. M. Sundar Rajan*, Associate Professor, Department of Industrial Control and Instrumentation, Faculty of Electrical and Computer Engineering, Institute of Technology, Arbaminch University, Ethiopia.

Email: msundarrajan84@gmail.com

Abraham Mesfin, Lecturer, Department of Industrial Control and Instrumentation, Faculty of Electrical and Computer Engineering, Institute of Technology, Arbaminch University, Ethiopia.

Satenaw Sando, Lecturer, Department of Computer Engineering, Faculty of Electrical and Computer Engineering, Institute of Technology, Arbaminch University, Ethiopia.

(C) The Authors. Published by Blue Eyes Intelligence Engineering and Sciences Publication (BEIESP). This is an open access article under the CC BY-NC-ND license (http://creativecommons.org/licenses/by-nc-nd/4.0/)

\section{INTRODUCTION}

loop control system with complete communication network and remote control system and sensors, actuators and controller signals transmitted over the network. A series of questions has been aroused by introducing the network especially the network-induced delay, so the control performance has affected seriously [1]. With the developing need for shared system and the improvement in the size of the applications, a conventional networked control systems(NCS) through wired correspondence systems to transmit the reports among conveying obstructs in the shut circle control system presents limitations. The utilization of remote correspondence systems gives significant advantages over their wired partners, which prompts discounted cost and solid system structures by lessening the cost of the establishment, change, and improve the system segments contrasted with their wired partner. This control system design is referred to as a remote NCS wherein the sensor and actuator speak with the controller by methods for a remote system. WNCS has been drawing the rising examination consideration and has been distinguishing a few applications in brilliant network, astute checking, and creating computerization. With its common advantages, WNCS encourages prospects on the assembling business [2]. Despite the fact that the Wireless Network Control System offers more focal points contrasted and the regular wired NCS, it is likewise unsurprising that the Wireless Network Control System is more helpless than wired Wireless Network Control System because of its normal "transparency" conduct from the remote system. The correspondence system plan for a Wireless Network Control System requires guaranteeing the working and strength of the control system, with the constrained battery assets of sensor hubs, despite the fact that the anomaly of remote transmissions and the common wireless medium [4].Comparing with the traditional control system, NCS has a few advantages including: less wiring, marked down cost, and greater adaptability and practicality of the model. Thus, NCS have been used comprehensively in the ongoing years in different spaces as like: modern control, process control, designing systems, aviation system, intelligent system, smaller scale networks, and teleoperation, to make reference to a couple. On the other hand, it changes out that the expansion of systems in dynamical system make new troubles to the whole system because of the development of anomalies. These include quantization mistakes, evolving delays, dropouts, and so on.

Published By:

Blue Eyes Intelligence Engineering DOI: $10.35940 /$ ijeat.D8983.049420

Journal Website: www.ijeat.org

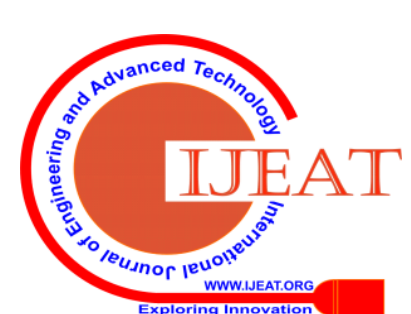


The shortcoming basically influence the exercises of the NCS by diminishing the working or creating insecurity. It is consequently basic to develop a proper unique portrayal of the NCS and plan successful controllers that accomplish stability under these conditions [5].

\section{SYSTEM DESCRIPTION}

\section{A. System Configuration}

An automotive control system comprises of a lot of devices that control at least one elements of the vehicle, for example, an electronic brake.

Current car control systems are networked control system(NCS) as appeared in Fig 1. A normal NCS contains the accompanying parts: Sensors to gain data from the physical condition. Actuators to play out the control orders. Controllers to give decision and orders. Correspondence organize for data trade between sensors, controllers and actuators. In a NCS, control and input signals are traded among the diverse system segments as data package through the communication network [9].

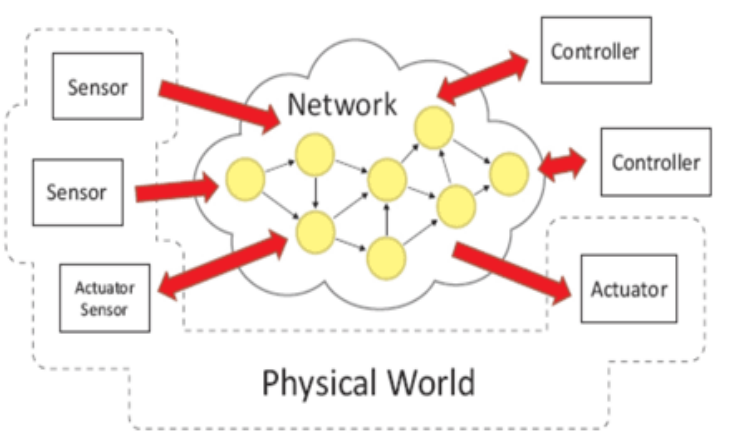

Fig 1. Network Control System

\section{B. Bandwidth Requirement Submission}

The inputs are sampled with sensors and then transferred to the controller by means of communication network. The outputs of the controller are transferred to the plant by means of network and then the closed-loop control is carried out by actuators. For the time being, the controller outputs are also dispatched to the scheduler as a basis for bandwidth assignment of communication network and sampling period decision of control system[12].

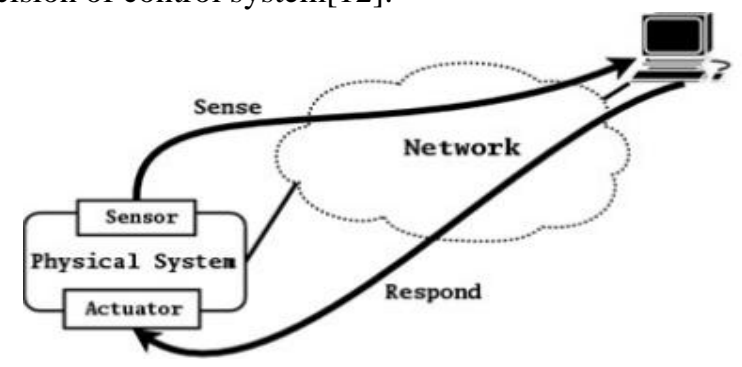

Fig 2. Information flow

The network congestion state is composed by scheduler. Then, the scheduler assigns the bandwidth for all channel depending on congestion state of network. In short, the scheduler method obtains QoS and QoP into account at the same time which can recognize control and scheduling code sign of NCS. In this sense, the NCS can be separated into control subsystem and network subsystem[8].Information flow is displayed as shown in Figure 2. The flow of information in network control system in the sensor controls the network and control bandwidth assignment. Delivery time is stable, bandwidth assignment control the packet and hence it is safe and performs good.

\section{Utility Function of NCS}

Exactly when the when is discretized by strategies for a couple of testing rates to execute a modernized controller over a system or PC, the control system response to dissipates diminishes appeared differently in relation to the steady shut circle type. The level of degradation relies upon the testing rate. To perceive the effect of inspecting rate on the disarray excusable capacity of a mechanized controller, we expect the RMS extent of the discrete time system plant state and its incessant time supplement. Typically, upgrading the inspecting rate will reduce the RMS of the structure's discrete plant state, where the industrious time system begins the lower bound of the RMS starts the lower bound of the RMS [6].

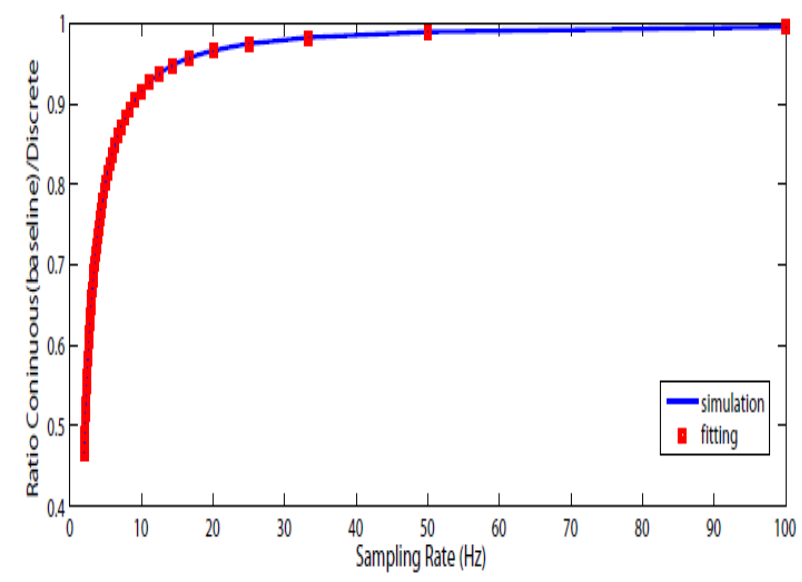

Fig 3. NCS for Utility Function

Authoritatively, how about we consider that the traffic from the controller to the plant has indistinguishable information way from the traffic from the plant to the controller. At that point a computerized control framework $f$ with test size pocket size and test time Ts will make a two way stream pace of pf $=2 \times$ pocket size/Ts to the system. We proclaim the utility capacity of framework $f$ as a component of rate $p f$ as beneath.

$$
U f(p f)=\frac{\text { RMScontinuous }}{\text { RMSdiscrete }(1 / T s)}
$$

Basically, the utility limit is made by taking a gander at the working of the discrete-time system with the consistent time structure. Using the persevering time control structure, we pick the limit of the control system to forgo disperses as redundant sound. We by then advancement to copy an equivalent system when a propelled use of the controller is used. This propelled execution relies upon the picked reviewing rate. Figure 2 is utility limit is basically picked by the measure of defilement of the structure response to repetitive noise to the closed loop system. [11].To approve our utility capacity definition, we accept the accompanying single-input-single yield (SISO) direct time invariant (LTI) framework, with no loss of sweeping statement and for ease.

Published By:

Blue Eyes Intelligence Engineering \& Sciences Publication 


\section{MECHANISM DESIGN CONCEPTS IN BANDWIDTH ASSIGNMENT}

\section{State-space Models Extending}

If we extend the state vector of all system (2) with a new state variable $b$ aimed at describing the bandwidth management dynamics, the new process can be modelled by

$\left[\begin{array}{l}x_{n+1} \\ b_{n+1}\end{array}\right]=\left[\begin{array}{cc}\varnothing(h) & \alpha \\ \beta & \gamma\end{array}\right]\left[\begin{array}{l}x_{n} \\ b_{n}\end{array}\right]+\left[\begin{array}{c}\Gamma(h) \\ \delta\end{array}\right] u_{n}$

$\alpha, \beta, \gamma, \delta$-matrices dimension

$x_{n}$ - original state variables

$b_{n}$ - dynamics bandwidth management

lattices measurement to be indicated, which will decide the connection of the transfer speed the board elements $b_{n}$ with the first state factors $x_{n}$, as further point by point in (3) and (4) separately.

$x_{n+1}=\Phi(h) x_{n}+\alpha b_{n}+\Gamma(h) u_{n}$

$b_{n+1}=\beta x_{n}+\gamma b_{n}+\delta u_{n}$

The un(input) is given by a new state feedback gain $\mathrm{L}=$ $\left[l_{x}, l_{b}\right]$ (as in (5)) designed according to (2), the extended model for the closed-loop system dynamics can be given by (4) and (5).

$u_{n}=\left[l_{x}, l_{b}\right]\left[\begin{array}{l}x_{n} \\ b_{n}\end{array}\right]$

\section{Customization of Model}

The last model (2) will allow every control loop configuration to obtain at all execution $b_{n+1}$, its next sampling period requirements.(bandwidth)

$\left[\begin{array}{l}x_{n+1} \\ b_{n+1}\end{array}\right]=\left[\begin{array}{cc}\varnothing(h) & 0 \\ \beta & \gamma\end{array}\right]\left[\begin{array}{l}x_{n} \\ b_{n}\end{array}\right]+\left[\begin{array}{c}\Gamma(h) \\ 0\end{array}\right] u_{n}$

$\alpha \simeq 0$, the dynamics of the original system are being modified. $\delta \simeq 0$, shut circle arrangement will be given by the state input control, will influence the development of $b_{n}$. Assume $\alpha=0, \delta=0 \beta=0$ and $\gamma=0$, then, for all $\mathrm{n}, \mathrm{bn}+1$ $=\mathrm{bn}$. That is, no dynamics are mentioned on the network bandwidth(equ (4)). $\beta=0$ and $\gamma<1$, where _( $(\cdot)$ is the spectral radius, bandwidth will reduce at all iteration, tending towards 0 , thus creating a system with no bandwidth, which makes no sense.

$\beta=0$ and $\gamma>1$, bandwidth will grows at all iteration, changing towards $\infty$, thus creating a system with infinite bandwidth, which is not realistic eventhough the expanded model (6) is flexible enough to specify different types of dynamics, if any linear combination of _ and does not satisfy the desired dynamics for the bandwidth management (new state variable), we can use nonlinear relations at the expenses of slightly increasing the complexity of the design of the control law, as we explain next[7].

\section{Dynamics Management of Bandwidth Specification} For all control loop, the bigger the error in Equation (7), the higher the bandwidth $b_{i, n}$ to be allocated.

$e_{i}=\left|x_{i}, n\right|$ set of networked control loops, a given parameter on the bandwidth usage factor should be kept (7), where Bg, the global bandwidth utilization factor, can be either the total existing bandwidth or a desired portion.

$\sum_{i=1}^{n} \cdot b_{i, n} \leq B_{g}$

For all control loop, we declare that at all closed loop function, the next sampling period $\mathrm{hn}+1$ bandwidth is a function of the exponential of the original state variables $x_{n}$. It considers both the current and global bandwidth utilization factors, $\mathrm{Bc}$ and $\mathrm{Bg}(8)$

$B_{a}=B_{g}-\left(B_{c}-\frac{m}{h_{n}}\right)=B_{g}-\left(B_{c}-b_{n}\right)$

bandwidth Ba can be calculated as in equation (9) and shortest possible sampling period $h_{\text {short }}$ that can be allocated to a control loop is mentioned by (10).

$h_{\text {short }}=\frac{m_{n}}{B_{a}}$

The next sampling period can be computed in Equation (11), $h_{\text {long }}$ and c are the longest feasible period and the criticalness, both to be defined for all control loop. The criticalness decides how quick a control loop will rise or reduce its period as per the error. More values for c will give more rapid changes in the sampling period.

$h_{n+1}=\left(h_{\text {long }}-h_{\text {short }}\right) e^{-c\left|x_{n}\right|}+h_{\text {short }}$

we get the model denoted in (12), where $\mathrm{h}_{\mathrm{l}}$ stands for $h_{\text {long }}$ and $\mathrm{h}_{\mathrm{s}}$ for $h_{\text {short }}$. Note that (12) is nonlinear.

$\left[\begin{array}{l}x_{n+1} \\ h_{n+1}\end{array}\right]=\left[\begin{array}{c}\Phi(h) \cdot x_{n} \\ \left(h_{l}-h_{s}\right) e^{-c\left|x_{n}\right|}+h_{s}\end{array}\right]+\left[\begin{array}{c}\Gamma(h) \\ 0\end{array}\right] u_{n}$

Therefore, the usage of the extended model needs the use of nonlinear control design techniques[10].

\section{Auctions}

Since all specialists need to battle for a limited transmission capacity, correspondences between these battling operators in a similar system can be demonstrated and depicted in a game-theoretic model. In this model, these specialists are estimated to be the players of a game, where all operator needs to pay a measure of cash to get transmission capacity with identified with its interest in a sale with different specialists during the task time frame TA. Our methodology needs that this sale is halfway directed by a passage. Regularly, run of the mill procedure for an operator to offer for a system data transfer capacity in a closeout are:

1. A specialist present a cost according to its data transmission requests.

2. The passage doles out data transfer capacities and tells all operators about the transmission capacities that will be determined.

3. The operator pays for the predefined data transfer capacity.

Regularly, all specialists doesn't have to pay for an overpricing data transfer capacity. Subsequently, in a sale, all specialists are to anticipate other operators' plays and select its own installment to expand its own benefit. In our methodology, all specialists fundamentally given a similar measure of cash,

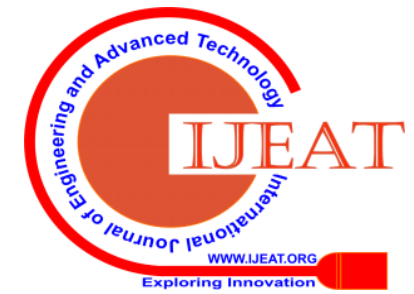


which is the most significant expense that an operator can pay. All specialists has an option to especially conclude how to utilize this cash in a task period TA and the measure of cash will be reset to $\mathrm{w}$ at the initiation of the following task period[6].

\section{SIMULATION RESULTS}

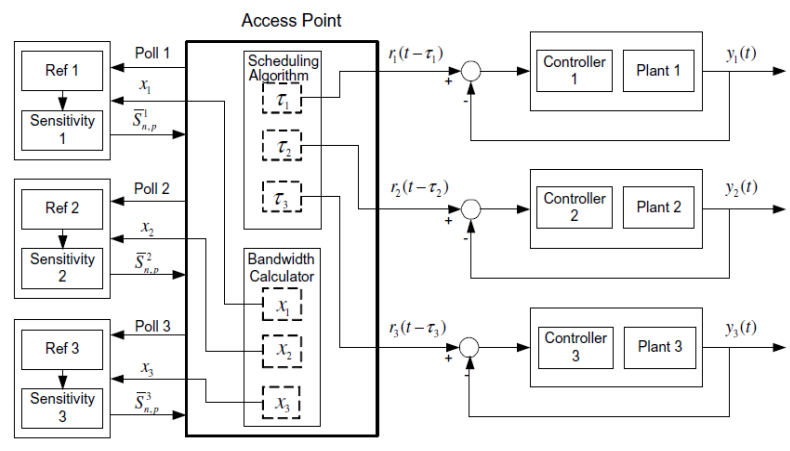

Fig.4 Simulation Model

MATLAB/SIMULINK is used for the simulation is dynamics bandwidth assignment to describe the proposed technique and check its efficiency. The simulation has 3 NCS, consists of 3 control agents and three motor plants with three control agents in a single wireless network with the bandwidth of 38,400 bits/s. In this simulation $\mathrm{TO}=0.40 \mathrm{~s}$, $\mathrm{TS}=0.004 \mathrm{~s}, \mathrm{TP}=0.02 \mathrm{~s}$ and the remaining of TO is TT. The assignment period TA is very small compared to TS so that TA can be considered to be zero. The entire system setting is shown in Figure 4. In this simulation a reference signal rk(t) will be deferred by $s_{k}$ according to the given bandwidth $\mathrm{X}_{\mathrm{k}}$ and a scheduling algorithm utilized[8.]

Table 1. Agent Average sensitivity

\begin{tabular}{|l|l|l|l|}
\hline Agents & First period & $\begin{array}{l}\text { Second } \\
\text { period }\end{array}$ & $\begin{array}{l}\text { Third } \\
\text { period }\end{array}$ \\
\hline $\mathbf{1}$ & 1.99 & 1.41 & 1.41 \\
\hline $\mathbf{2}$ & 2.74 & 1.59 & 2.74 \\
\hline $\mathbf{3}$ & 1.4 & 1.19 & 1.4 \\
\hline
\end{tabular}

\section{Agent Average sensitivity}

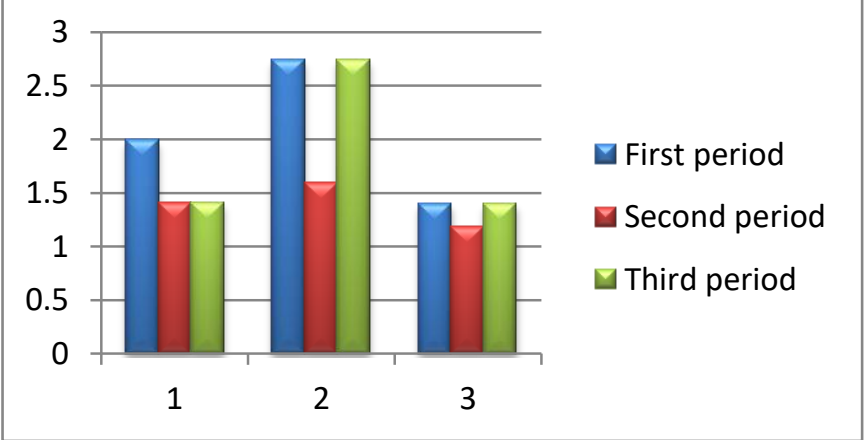

Fig 5. Agent Average sensitivity

From above table 1 is Operator Average affectability, a little data transfer capacity is likely important to send the suggestion for the specialist 3 . Consequently, the absolute execution case is genuinely most exceedingly awful. With the task dependent on affectability component, an operator is given a relegated transmission capacity with identified with its normal affectability in Table 1(Figure 5) without thinking about the activities of different specialists. Regardless of whether the particularized introduction of a specialist in not many periods might be more, the all-out presentation contrasted with different systems may not be acceptable as shown in Table 3(Figure 9).

Table 2 Sensitivity Optimizing Auction-Based Dynamic Bandwidth Assignment

\begin{tabular}{|l|l|l|l|}
\hline Agents & First period & $\begin{array}{l}\text { Second } \\
\text { period }\end{array}$ & $\begin{array}{l}\text { Third } \\
\text { period }\end{array}$ \\
\hline $\mathbf{1}$ & 0.0323 & 0.0153 & 0.0150 \\
\cline { 2 - 4 } & 0.0315 & 0.0131 & 0.0174 \\
\cline { 2 - 4 } & 0.0224 & 0.0101 & 0.0544 \\
\cline { 2 - 4 } & 0.0359 & 0.0109 & 0.0165 \\
\hline \multirow{4}{*}{} & 0.0584 & 0.0124 & 0.0585 \\
\cline { 2 - 4 } & 0.0441 & 0.0096 & 0.0354 \\
\cline { 2 - 4 } & 0.0176 & 0.0069 & 0.0146 \\
\cline { 2 - 4 } & 0.0364 & 0.0094 & 0.0368 \\
\hline \multirow{5}{*}{} & 0.0142 & 0.0006 & 0.0143 \\
\cline { 2 - 4 } & 0.0216 & 0.0004 & 0.0171 \\
\cline { 2 - 4 } & 0.2300 & 0.0045 & 0.0544 \\
\cline { 2 - 4 } & 0.0165 & 0.0001 & 0.0165 \\
\hline
\end{tabular}

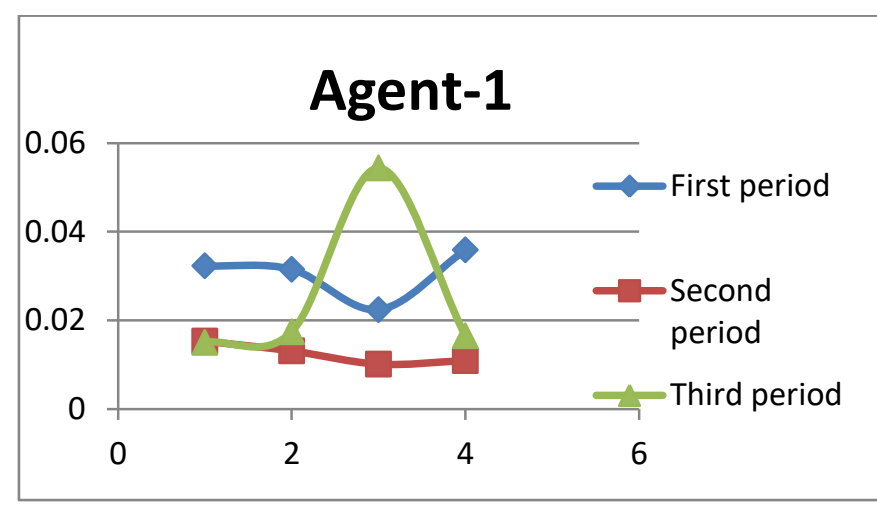

Fig.6 Agent-1

Table 2 is particularized execution estimates rises when operators are given little amount of transfer speeds. The equivalent data transfer capacity task technique consistently doles out an equivalent transmission capacity to all specialists. Consequently, not many operators are given unnecessary transfer speed contrasted with others. A particularized exhibition measure all operators is shown in Figure 6, 7, 8. 


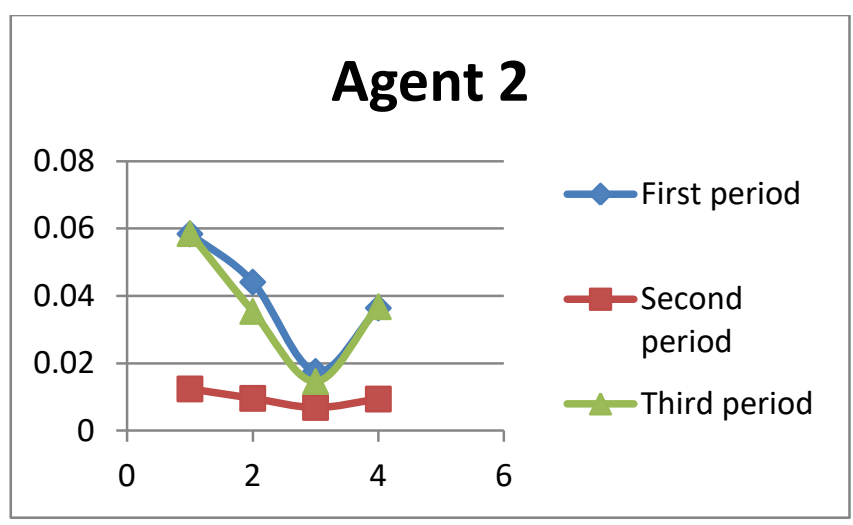

Fig.7 Agent-2

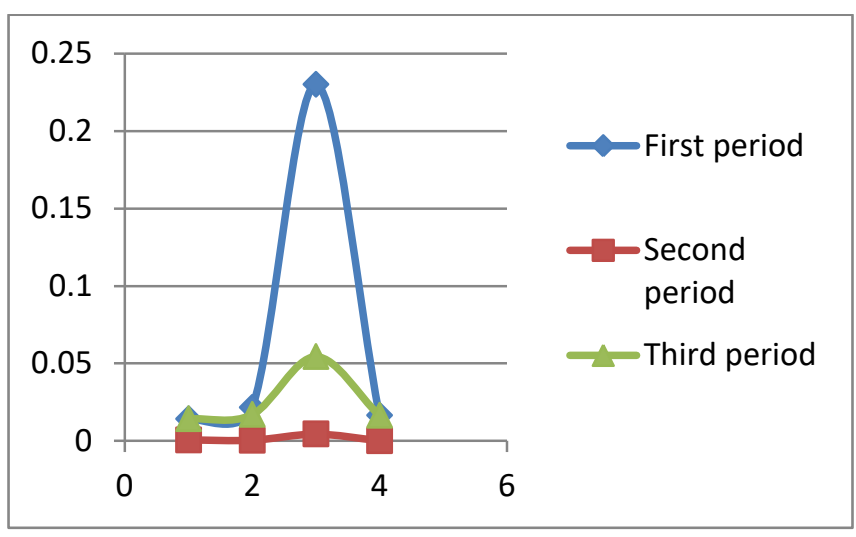

Fig.8 Agent-3

Even if the particularized performance of an agent in few time duration may be more, the total performance is analyzed with the other methods and are not be most favorable as shown in Table 3(Figure 9).

Table 3. Entire performance measures in $\mathbf{3}$ assignment periods

\begin{tabular}{|l|l|l|l|}
\hline $\begin{array}{l}\text { Assignment } \\
\text { method }\end{array}$ & $\begin{array}{l}\text { First } \\
\text { period }\end{array}$ & $\begin{array}{l}\text { Second } \\
\text { period }\end{array}$ & $\begin{array}{l}\text { Third } \\
\text { period }\end{array}$ \\
\hline $\begin{array}{l}\text { Equal } \\
\text { bandwidth } \\
\text { assignment }\end{array}$ & 0.1049 & 0.0305 & 0.0918 \\
\hline $\begin{array}{l}\text { Optimizing } \\
\text { utilities }\end{array}$ & 0.2710 & 0.0209 & 0.1254 \\
\hline $\begin{array}{l}\text { Sensitivity } \\
\text { based } \\
\text { assignment }\end{array}$ & 0.0972 & 0.0242 & 0.0719 \\
\hline $\begin{array}{l}\text { Auction-based } \\
\text { dynamic } \\
\text { assignment }\end{array}$ & 0.0908 & 0.0221 & 0.0718 \\
\hline
\end{tabular}
schemes as per the simulation outputs.

The total performance is superior than the functioning in the earlier simulation because of some noises. The values are tabulated in table 4. Conversely, the auction-based dynamic assignment still offers the good performances between other

Table 4. all agents Entire performance measures in three assignment time intervals

\begin{tabular}{|l|l|l|l|}
\hline $\begin{array}{l}\text { Assignment } \\
\text { method }\end{array}$ & $\begin{array}{l}\text { First } \\
\text { period }\end{array}$ & $\begin{array}{l}\text { Second } \\
\text { period }\end{array}$ & $\begin{array}{l}\text { Third } \\
\text { period }\end{array}$ \\
\hline $\begin{array}{l}\text { Equal } \\
\text { bandwidth } \\
\text { assignment }\end{array}$ & 0.1051 & 0.0640 & 0.1109 \\
\hline $\begin{array}{l}\text { Optimizing } \\
\text { utilities }\end{array}$ & 0.2588 & 0.0604 & 0.1863 \\
\hline $\begin{array}{l}\text { Sensitivity } \\
\text { based } \\
\text { assignment }\end{array}$ & 0.1104 & 0.0556 & 0.1101 \\
\hline $\begin{array}{l}\text { Auction-based } \\
\text { dynamic } \\
\text { assignment }\end{array}$ & 0.0989 & 0.0454 & 0.1084 \\
\hline
\end{tabular}

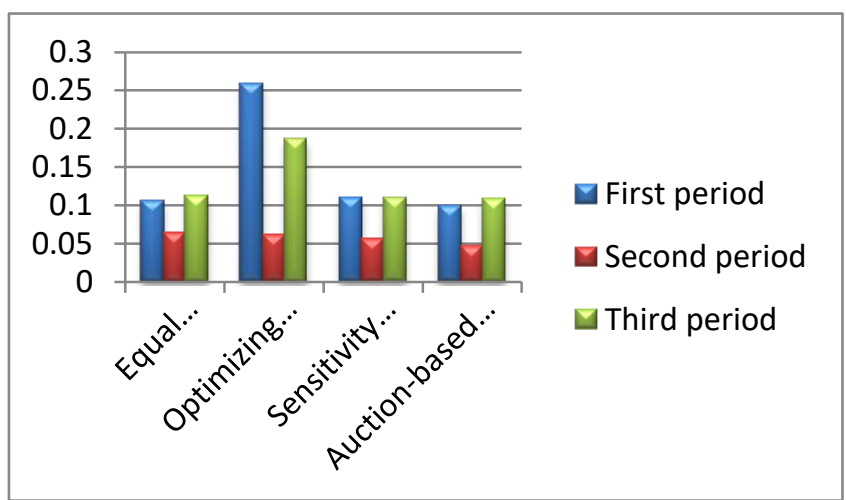

Fig.10 All Agents Entire Performance Measures

\section{CONCLUSION}

This paper proposes an auction-based dynamic assignment methods to assign bandwidth for open-loop NCS. Average output sensitivities of NCS is utilized to denote bandwidth necessity in the mode of an utility function. The suggested method produces proof that real bandwidth requirement is the major plan and the surpass bandwidth necessity cannot 
offer the advanced utility than the real bandwidth necessity, even though the assigned bandwidth of over request is more than assigned bandwidth of real strategy. This system calculate the entire performance all 3 agents and Entire performance measures in 3 assignment periods, Average sensitivity, Sensitivity Optimizing Auction-Based Dynamic Bandwidth Assignment. NCSs have been widespread and widely applied for several years due to their various benefits and extensive applications.

\section{REFERENCE}

1 Sun Chao, Zhou Ying, Dai Junxiang, Lin Jinxing, " Networked Control System Time Delay Compensation Based on Improved Implicit GPC", 36th Chinese Control Conference, 2017.

2 Yuan Yuan, Huanhuan Yuan, Daniel W. C. Ho, " Resilient Control of Wireless Networked Control System Under Denial-of-Service Attacks: A Cross-Layer Design Approach", IEEE TRANSACTIONS ON CYBERNETICS, 2018.

3 R. Munoz,N. Yoshikane, R. Vilalta, J. M. Fabrega, L. Rodriguez, D. Soma,S. Beppu, S. Sumita, R. Casellas, R. Martinez, T. Tsuritani, I. Morita, " Adaptive software defined networking control of space division multiplexing super-channels exploiting the spatial-mode dimension", Journal of Optical Communications and Networking, 2020.

4 Magdi S. Mahmoud and Mutaz M. Hamdan, " Fundamental Issues in Networked Control Systems", IEEE/CAA Journal Of Automatica Sinica, Vol. 5, No. 5, September 2018.

5 Yang Tao, Huang Wei, Zhao Yixin, " A Dynamic Assignment Strategy of Bandwidth of Networked Control Systems with Bandwidth Constraints", IEEE, 2016.

6 Tomer Gafni ; Kobi Cohen, " A Distributed Stable Strategy Learning Algorithm for Multi-User Dynamic Spectrum Access", 57th Annual Allerton Conference on Communication, Control, and Computing (Allerton),2019.

7 Wang Zhi-wen ; Cheng Yang, " Bandwidth Assignment Strategy of Networked Control System under Denial-of-Service Attack", 4th Annual International Conference on Network and Information Systems for Computers (ICNISC), 2018.

8 Amin Nazarzadeh ; Mohsen Montazeri, " Event-based Control and Scheduling Co-Design in Networked Control Systems with Bandwidth Limitation", 27th Iranian Conference on Electrical Engineering (ICEE), 2019.

9 Zhiwen Wang ; Hongtao Sun, " Bandwidth scheduling of networked control system based on time varying sampling period", Proceedings of the 32nd Chinese Control Conference, 2013.

10 Suwatchai Kamonsantiroj, Luepol Pipanmaekaporn, " Decentralized Auction-Based Bandwidth Assignment in A Wireless Networked Control Systems", 3rd International Conference on Advanced Applied Informatics, 2014.

11 Indra Sakti ; Dicky Rianto Prajitno, " Experiment of Networked Control System (NCS) using network emulator", 3rd International Conference on Information Technology, Computer, and Electrical Engineering (ICITACEE), 2016.

12 Xianming Fang ; Feng Du ; Yinqing Tang ; Bing Ma ; Chunrong Huang, "A study of deadband scheduling based on sensor node and controller node in NCS", The 27th Chinese Control and Decision Conference, 2015

13 Dr.M.Sundar Rajan, Scheduling in Networked Control System for Monitoring and Protection Applications in Sensor Nodes", CiiT International Journal of Networking and Communication Engineering, Vol.8, No.10, Nov-Dec 2016.

\section{AUTHORS PROFILE}

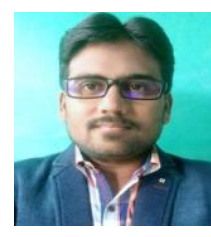

Dr.M.Sundar Rajan was born in Vellore, India in 1984 .He received B.E., M.Tech and Ph.D Degree in Electrical Engineering in 2005, 2007 and 2013 respectively. He is working as an Associate Professor in Department of Industrial Control and Instrumentation, Faculty of Electrical and Computer Engineering in Arbaminch University, Ethiopia. He is having more than 12 years of teaching and 4years of research experience.
He published more than 10 papers in International Journals and One Patent. He has received Excellence in Teaching and Research award. He is member of professional organizations such as IEEE, IAENG, IACSIT, SDIWC, SMIRED and Editorial Member for several International Journals. His research areas include Control Systems, Wireless Sensor Networks and Image Processing.

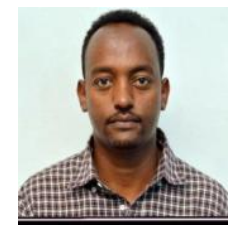

Abraham Mesfin was born in Addis Ababa, Ethiopia, in 1988. He received the B.Sc. degree in Electrical Engineering from Haramaya University in 2010 and the M.Sc. degree in Control Engineering in 2018 from Addis Ababa University, Ethiopia. He is working as Lecturer and Chair Head for Department of Industrial Control and Instrumentation, Faculty of Electrical and Computer Engineering Arbaminch University, Ethiopia. His current research interests include Design, Analysis and Control of Power Electronic Systems, AC Motor Drive and Sensorless Control.

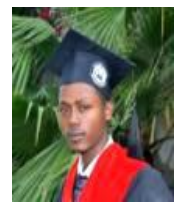

Satenew Sando was born in Ethiopia in 1988. He received the B.Sc. degree in Electrical Engineering from Arbaminch University in 2011 and the M.Sc. degree in Microelectronics in 2014 from Addis Ababa University, Ethiopia. He is working as Lecturer and Dean for Faculty of Electrical and Computer Engineering Arbaminch University, Ethiopia. His current research interests include Microelectronics, Embedded Systems and Wireless Sensor Networks. 